\title{
Teaching K-12 teachers and students about nanoscale science through microscopy
}

\section{Nancy Healy}

Nancy Healy, "Teaching K-12 teachers and students about nanoscale science through microscopy," Proc. SPIE 9236, Scanning Microscopies 2014, 92360P (16 September 2014); doi: 10.1117/12.2074629

SPIE Event: SPIE Scanning Microscopies, 2014, Monterey, California, United States 


\title{
Teaching K-12 teachers and students about nanoscale science through microscopy
}

\author{
Nancy Healy \\ Georgia Institute of Technology - Institute for Electronics and Nanotechnology \& NNIN, 791 \\ Atlantic Dr. NW, Atlanta, GA, USA 30332
}

\begin{abstract}
The National Nanotechnology Infrastructure Network (NNIN) is an integrated partnership of 14 universities across the US funded by NSF to support nanoscale researchers. NNIN's education and outreach programs are large and varied and includes outreach to the K-12 community in the form of professional development workshops and school programs. Two important components of nanoscale science education are understanding size and scale and the tools used in nanoscale science and engineering (NSE). As part of our K-12 endeavors, we educate K-12 students and teachers about the tools of nanoscience by providing experiences with the Hitachi TM 3000 tabletop Scanning Electron Microscope (SEM). There are three of these across the network that are used in education and outreach. This paper will discuss approaches we use to engage the K-12 community at NNIN's site at Georgia Institute of Technology to understand size and scale and the applications of a variety of microscopes to demonstrate the imaging capabilities of these to see both the micro and nano scales. We not only use the tabletop SEM but also include USB digital microscopes, a Keyence VHX600 Digital Microscope, and even a small lens used with smart phones. The goal of this outreach is to educate students as well as teachers about the capabilities of the various instruments and their importance at different size scales.
\end{abstract}

Keywords: nanotechnology education, size and scale, microscopy in STEM education

\section{INTRODUCTION}

Nanoscale Science and Engineering (NSE) is a fast growing area of science and engineering that crosses all discipline boundaries. Several studies have stressed the importance of including nanoscale concepts and topics in the K-12 curriculum. The National Nanotechnology Infrastructure Network (NNIN) is a NSF-funded program which supports nanoscience researchers by providing state-of-the-art facilities, support, and resources. The NNIN is a partnership of 14 U.S. universities (http://www.nnin.org) which provide researcher support. NNIN also has a networked education program which has a variety of offerings for school-age children through adult professionals. Our mission is to address the explosive growth of nanotechnology and its growing need for a skilled workforce and informed public by offering education and training to individuals. We provide resources, programs, and materials to enhance an individual's knowledge of nanotechnology and its application to real-world issues. We believe that a strong U.S. economy requires a STEM-literate workforce ready to meet the technological challenges of a nano-enabled economy as well as an informed citizenry that supports continued and safe growth of nanotechnologies.

As part of our efforts to encourage and develop a STEM-ready workforce, we provide programs for K-12 teachers and students. This paper will focus on two aspects of our K-12 education programs that focuses on educating K-12 teachers and students about size and scale and the tools of nanoscale science and engineering, in particular SEMs.

\section{EXPLORING SIZE AND SCALE}

The concept of scale is one of the four unifying themes identified by AAAS's Project $2061^{1}$ and is considered central to most scientific disciplines. Research on size and scale indicates that both students and adults have difficulty in conceptualizing the nanoscale; that relative scale is easier to understand than absolute scale, that we use objects to conceptualize scale; and we use ourselves as our reference point for relative scale. ${ }^{2-4}$ Tretter et $\mathrm{al}^{4} \mathrm{determined} \mathrm{that}$ accuracy in placing objects along a size scale decreased as the objects moved from the visible range to the scale where visibility is limited for the unaided eye. Yet for nanoscale science, it is only through specialized tools and instruments that we can see objects at this small scale. Thus it becomes important in teaching NSE that one not only help individuals

Scanning Microscopies 2014, edited by Michael T. Postek, Dale E. Newbury, S. Frank Platek, Tim K. Maugel, Proc. of SPIE Vol. 9236, 92360P · (c) 2014 SPIE · CCC code: 0277-786X/14/\$18 - doi: 10.1117/12.2074629 
understand the concept of size and scale but also include experiences with the tools for seeing and manipulating objects and materials at the nanoscale.

After ten years of providing teacher professional development and student outreach programs, NNIN Education and Outreach (E\&O) knows that to understand NSE you must begin with an understanding of size and scale. This was further confirmed with our collaboration on the Mid-Continent for Research Education and Learning's (McREL) NanoTeach project ${ }^{5}$. Teacher participants in this five year NSF-funded project confirmed that the first step for including NSE in the classroom must begin with an understanding of size and scale. Many of the NanoTeach participants created scale lines or "size and scale" bulletin boards around their classrooms to have students place classroom topics (current science teaching focus) into the four scales of measurement (discussed below). They indicated that this became a powerful tool in helping students understand what world of measurement science topics/objects being studied in the classroom should be placed in.

Our work with K-12 teachers and students is based on the concepts detailed in the book Big Ideas of Nanoscale Science and Engineering: A Guidebook for Secondary Teachers ${ }^{6}$. We chose to use these big ideas as they provide a foundation of the important concepts in nanotechnology and they are directly related to science curriculum. Importantly, each Big Idea comes with a series of learning goals and misconceptions which help guide approaches to instruction. There are nine big ideas of which this paper will focus on two - Size and Scale and Tools and Instrumentation.

We begin to develop the concept of size and scale by discussing that there are four worlds or scales of measurement macro, micro, nano, and atomic. We use the commonly held definition of the nanoscale to be 1-100 nanometers in one direction $^{7-8}$. Teachers and students explore their understanding of scale by performing a variety of activities which include size sorting of objects (printed on cards) as well as working with scale interactives available on-line. For the size sorting activity, we use either one developed by NanoSense ${ }^{9}$ or one available on the NNIN education portal ${ }^{10}$. We often use the Nanosense activity because one of the object cards is the visible light spectrum which ties in directly to our activities focused on microscopes. Figure 1. shows a student sorting the NanoSense object cards from largest to smallest. Regardless of the age or educational level of the participants, nearly all have difficulty as the objects move from the micro to the nanoscale. This observation confirms the results reported by Tretter et $\mathrm{al}^{4}$. Interestingly, we have found that some students are be able to tell us that a molecule is made up of atoms and that atoms are made up of electrons, neutrons, and protons but when it comes to sorting these objects on the cards they will put the nucleus of an atom to be larger than a molecule. This activity provides an excellent opportunity to discuss the size of objects between the nano and atomic scales and to help students with misconceptions with objects at these scales.

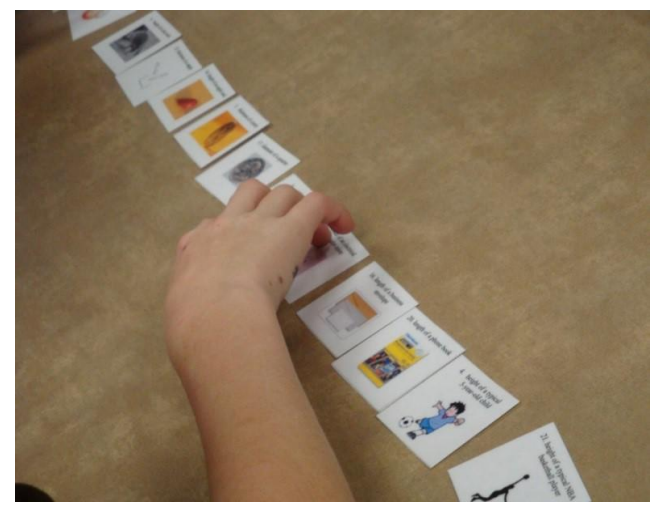

Figure 1. K-12 student sorting images of objects in terms of relative size.

In addition to using object sorting on a size scale, there are numerous online sites that allow exploration of size and scale from the universe down to the atomic scale. Research has shown that using videos can positively impact student understanding of scale ${ }^{12}$. In Jones et al study ${ }^{11}$, The Powers of Ten video ${ }^{12}$ was used with middle school students and the study reported that the students had improved understanding of the powers of ten and scale post-viewing of the video. In turn, one would expect that student interaction with online scaling activities would also improve understanding of scale. Table 1 below provides a list of several websites that GT-NNIN has used with the K-12 community to explore 
interactive size scales. The table represents not an all-inclusive list but a sampling of interactive scale activities that can be found on the web.

Table 1. List of some resources useful for exploring size and scale.

\begin{tabular}{|l|l|}
\hline Description & Website \\
\hline $\begin{array}{l}\text { Multi-scale graph of common } \\
\text { object }\end{array}$ & http://www.mcrel.org/nanoleap/multimedia/Nanosize_me.swf \\
\hline $\begin{array}{l}\text { Interactive and film based on } \\
\text { the work of Charles and Ray } \\
\text { Eames }\end{array}$ & http://www.powersof10.com/ \\
\hline $\begin{array}{l}\text { Interactive scale and Powers } \\
\text { of Ten }\end{array}$ & http://scaleofuniverse.com \\
\hline $\begin{array}{l}\text { Animation on microscopy } \\
\text { and scale }\end{array}$ & http://www.cneu.psu.edu/edToolsActivities.html \\
\hline Animation on the size of cells & http://www.cellsalive.com/howbig.htm \\
\hline $\begin{array}{l}\text { Animated trip through many } \\
\text { scales }\end{array}$ & http://www.nanoreisen.de/english/index.html \\
\hline $\begin{array}{l}\text { Images from the universe to } \\
\text { the nanoscale }\end{array}$ & http://micro.magnet.fsu.edu/primer/java/scienceopticsu/powersof10/ \\
\hline $\begin{array}{l}\text { Card game using the Powers } \\
\text { of Tech }\end{array}$ & $\begin{array}{l}\text { http://www.nisenet.org/catalog/programs/exploring_size_- } \\
\text { powers_ten_game_nanodays_2011 }\end{array}$ \\
\hline $\begin{array}{l}\text { Scale Ladder } \\
\text { chart/ }\end{array}$ \\
\hline Scale of Things chart \\
\hline
\end{tabular}

\section{EXPLORING THE TOOLS OF NANOTECHNOLOGY - SEMS}

One of the learning goals of the Big Idea Size and Scale is that there are "some worlds too small to be seen with the naked eye, including the micro-, nano- and atomic and molecular worlds; each of these contains unique representative objects that help define the scale represented by the world." This learning goal serves as a natural bridge to the Big Idea Tools and Instrumentation. It allows us to discuss the tools and instruments used in NSE to see and manipulate objects at these very small scales. The development of such tools as Scanning Tunneling Microscopes (STM). Atomic Force Microscopes (AFM), and recent advances in SEM technology have allowed scientists and engineers to develop new levels of understanding of matter. With these tools, we are now able to measure, examine, manipulate, and fabricate at the nanoscale. Exploring these tools is a great way to educate students and teachers on how tools and instruments are key to advances in science and engineering and in particular nanotechnology.

Our approach for exploring microscopes is to allow teachers and students to experience a variety of magnifying instruments. The instruments that are used depends on the age of the participants. For very young students, we have developed a lesson "Taking a Closer Look" "which uses a microscope made from hand-held magnifiers and demonstrates to them how we use tools to see objects and to see greater detail of objects by using such devices. The purpose of this grades 2-5 lesson is in order for students to understand their world they often need to use tools to gather information. Some tools will help them see parts of their surrounding world that they would otherwise miss. The tools in the lesson they use include magnifying glasses and microscopes. Importantly the lesson allows these young students an opportunity to learn how microscopes and lenses make small objects in our world visible. 

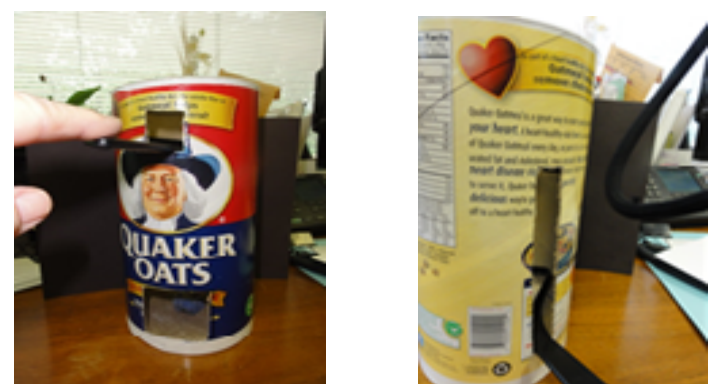

Figure 2. Image of "microscope" made using magnifying lenses and oatmeal containers - based on the design in Van Cleave, $1993^{14}$

As we move up in age, we can begin to examine and discuss the electromagnetic spectrum and that the visible light range is 400-700 nanometers. We explain that nanoscale objects are below the visible light range and therefore cannot be seen with optical microscopes and that other forms of radiation are used to explore objects outside of the visible light range. To see objects below the visible light range, we must use special instruments such as Scanning Tunneling Microscopes, Atomic Force Microscopes, and Scanning Electron Microscopes. Typical activities for this "Big Idea" include the use of several different imaging devices -- simple hand lens, USB digital scopes, micro phone lens, optical microscopes, a Keyence VHX-600 Digital Microscope, SEM, and AFM. The learning objectives are for students and teachers to understand that different imaging tools are suitable for certain objects, that not all objects can be seen with commonly used microscopes and lenses, and that the resolution of the instruments varies allowing for different amounts of information that can obtained. The concept that light cannot be used to image all objects is difficult for many students to understand. Using a variety of imaging devices helps students to understand capabilities and limitations of devices with various objects. For example, a classroom visit with the Hitachi TM 3000 tabletop SEM will include us bringing some samples for students to examine as well as asking the class to bring samples. We provide hand lenses, USB digital microscopes, and the SEM for viewing the specimens. They are then able to see which instruments are needed for which objects.

Choosing the right tool for the object to be examined is an important concept to understand in science and engineering. For example, examining a penny with a hand lens or optical microscope provides sufficient magnification to see surface features. Using an SEM to view the penny is possible but one would only use very low magnification which can be obtained with the hand lens or optical scope. Conversely, examining pollen grains is better suited for the SEM as we can distinguish surface features not visible with a hand lens or optical microscope. By providing students and teachers with a variety of magnifying tools and a variety of objects to examine, they are able to determine which tool is suitable for which object. They also develop an understanding of the capabilities and limitations of the various instruments used. This allows us to educate individuals on the capabilities of imaging devices and to develop an understanding of the importance of using the correct tool to investigate objects, materials, and/or phenomena. Gaining knowledge of the right tool to use ties back to the importance of understanding size and scale as one must consider the size of the object, material, or phenomena to be investigated to determine which tool will be best to use in examining that object. This in turns leads into a discussion of the importance of tools and instruments in exploring the nanoscale for without these tools we would not be able to make the gains we have in NSE. A question we often ask program participants is how do you make things at the nanoscale and how do you know that you have actually made something that small. AFM, STM, and SEM allow scientists and engineers to manipulate, fabricate, and visualize matter at the nanoscale.
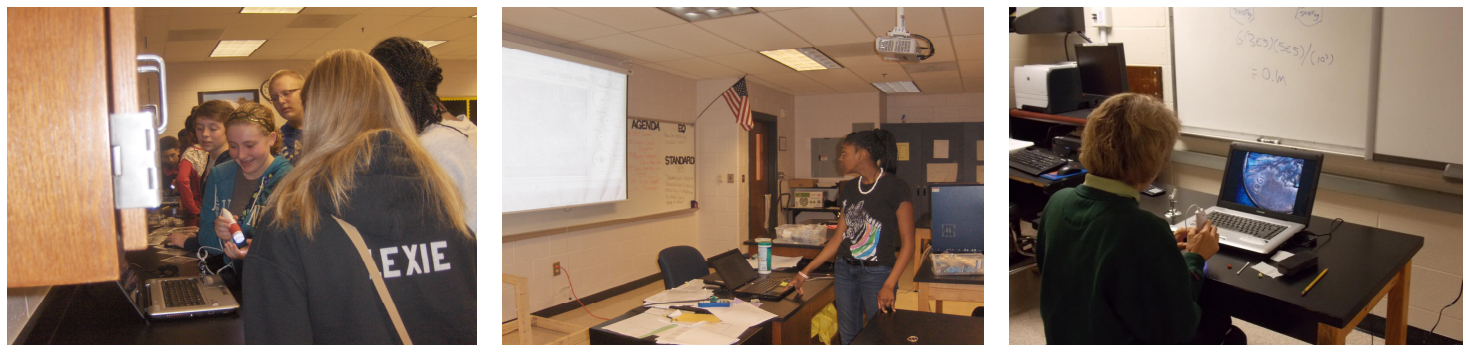

Figure 3. Images of students using a USB digital scope (A\&C) and the Hitachi TM3000 tabletop SEM. 
Why is an understanding of tools and instrumentaion important? First, it is our belief that students should be exposed to the tools of science and engineering at an early age. We have used the NNIN lesson The Right Tool for the Job ${ }^{15}$ with students as young as kindergarten. While we do not expect them to learn about microscopy at this age, we hope to lay the foundation that they need the right tool to perform a particular task. As we progress through the grades, we introduce various types of microscopes as well as information on nanoscale objects, devices, and materials. Our approach is a continuum of educating students about microscopy culminating with the use of the tabletop SEM and our NanoSurf EasyScan AFM by NanoScience Instruments. We hope by exposing students to a variety of instruments that they will develop an understanding of the impact microscopy has on science and engineering.

Another important objective is that we want students to understand that nanoscale objects are too small to be seen with the unaided eye and that they are too small to be seen with optical microscopes. Even with middle school students, it is possible to discuss that SEMs and AFMs use electrons and forces to discern the objects too small to be seen with conventional optical microscopes. The Next Generation Science Standards for middle school students (Structure and Property of Matter) include student understanding of the composition of molecules at the atomic level. Thus a discussion of the use of electrons and atomic forces to discern objects is suitable at these grade levels. To further enhance their understanding of an SEM, we provide students with either a hard copy or link to Hitachi's Science is Fun All about electron microscopes ${ }^{16}$.

One other objective of using microscopes in STEM/Nano outreach is to excite students about science and engineering and in particular NSE. A primary goal of NNIN Education and Outreach is to encourage students to pursue education paths in science or engineering to help in the development of a STEM and nano-ready workforce. NSF $^{7}$ estimates that by the year 2015 there will be a need for nearly 1 million US workers in nanotechnology related fields. We have found that students are very enthusiastic when allowed to explore the worlds seen by simple USB scopes but that this enthusiasm increases when allowed to use an SEM. We are fortunate to have three SEMs across the NNIN that can be used either onsite or offsite at schools and science centers. At the National Science Teachers Association meetings, NNIN partners with Hitachi, HTA to have a TM3000 at our exhibit booth and we have a daily "picture of the day" contest. This involves attendees bringing their own sample(s) to scan with a prize provided by Hitachi, HTA at the end of the day for the best image. We provide the contest participants all of the images captured over the three days so that they can use them in their classroom. They also learn about Hitachi, HTA's program to loan a tabletop electron microscope to school districts, science museums, community and technical colleges, and universities.

This is just one approach we have used to incorporate microscopes into our education and outreach programs. Another activity that we use is to have students create their own Powers of Ten for objects of their own choosing. Patterned after the SEM activity developed by Duke University's TIP program ${ }^{17}$, they develop a poster or PowerPoint which describes the resolution possible at each magnification level used. When doing outreach where it is not suitable to use the SEM (outdoor events or large science festivals, for example), we use an SEM matching "game". For this, we bring objects, printed SEM images of the objects, and a USB digital scope or hand lens. Event attendees are then asked to match the image to the correct object. We have signage about how the SEM images were created which we use to educate about the use of SEMs. It is a fun and easy way to include SEMs in outreach where the use of instruments is not feasible. We also encourage teachers to explore remote access to SEMs available at several universities such as at Pennsylvania State University and Lehigh University, among others.

\section{SUMMARY}

This paper provides a short overview of approaches used by the NNIN site at Georgia Tech to use size and scale activities and various microscopes to educate and excite students about STEM and nanoscale science and engineering. Emphasized here is the importance of connecting the size of objects to the instruments used to see these objects. Using the Big Ideas of Nanoscale Science allows us to develop activities that address nanoscale learning goals as well as student misconceptions about size and scale as well as about tools and instruments. In particular, students must learn that nanoscale objects fall below the range of visible light and must be viewed by special instruments such as SEMs and AFMs. Through our activities, we hope that program participants understand that special instruments are needed to visualize, manipulate, and analyze materials at the nanoscale. In addition, we hope to excite students about the world seen through microscopes. 


\section{References}

[1] American Association for the Advancement of Science, Project 2061 Benchmarks for Science Literacy, Oxford University Press, 418 pages New York (1993).

[2] Tretter, T.R., Jones, G.M., Andre, T., Negishi, A., and Minogue, J., "Conceptual boundaries and distances: Students' and experts' concepts of the scale of scientific phenomena," J. Res. in Science Teaching 43(3), 282-319, (2006).

[3] Tretter, T.R., Jones, G.M., and Minogue, J., "Accuracy of scale conceptions in science: Mental maneuverings across many orders of spatial magnitude," J. Res. in Science Teaching 43 (10), 1061-1085, (2006).

[4] Tretter, T.R., Jones, G.M., and Falvo, M., "Impact of introductory nanoscience course on college freshmen's conceptions of spaial scale," J. of Nano Ed., 2, 53-66.

[5] NanoTeach, accessed at: http://www.mcrel.org/NanoTeach/

[6] Stevens, S.Y., Sutherland, L.M., and Krajcik, The Big Ideas of Nanoscale Science and Engineering: A Guidebook for Secondary Teachers, NSTA Press, Arlaington, VA., 203 pages, (2009).

[7] Roco, M. "Converging Science and Technology at the Nanoscale: Opportunities for Education and Training", Nature Biotechnology, 21 (10), 1-3, (2003).

[8] National Nanotechnology Initiative accessed at: http://www.nano.gov/nanotech-101

[9] NanoSense Size Matters Lesson 2 Size and Scale, Accessed at: http://nanosense.sri.com/activities/sizematters/sizeandscale/SM_Lesson2Student.pdf)

[10] NNIN Size and Scale - Learning about measurement, accessed at: http://www.nnin.org/education-training/k-12teachers/nanotechnology-curriculum-materials/size-and-scale-learning-about

[11] Jones, G,M., Taylor, A., Minogue, J., Broadwell, B., Wiebe, E., and Carter, G., "Understanding scale: Powers of Ten," J. Sci. Ed. and Technology, 16 (2), 191-202, (2007).

[12] The Power of Ten, accessed at: http://powersof10.com.

[13] NNIN Taking a Closer Look at Objects, accessed at: http://nnin.org/education-training/k-12teachers/nanotechnology-curriculum-materials/taking-closer-look-objects

[14] Jan VanCleave, Microscopes and Magnifying Lenses, John Wiley and Sons, New York, 100 pages, (1993).

[15] NNIN Right Tool for the Job, accessed at: http://nnin.org/education-training/k-12-teachers/nanotechnologycurriculum-materials/right-tool-job.

[16] Hitachi,HTA, Science is Fun, accessed at: http://www.inspirestemeducation.us/tools/science-is-fun/

[17] Donovan Leonard of Appalachian State University and Duke University's TIP program accessed at: $\mathrm{http} / /$ www.inspirestemeducation.us/tools/sem-appalachian-state-university/ 